\title{
A Study of Executive Function in Patients with Chronic Kidney Disease before and after a Single Session of Hemodialysis
}

\author{
Vasantmeghna S. Murthy ${ }^{1}$ Vedant S. Shukla \\ ${ }^{1}$ Department of Psychiatry, Krishna Institute of Medical Sciences, \\ Karad, Maharashtra, India
}

J Neurosci Rural Pract 2020;11:250-255

\begin{abstract}
Address for correspondence Vasantmeghna S. Murthy, MD, Department of Psychiatry, Krishna Institute of Medical Sciences, OPD - 22, Karad, Satara, Maharashtra 415110, India (e-mail: svasantmeghna@gmail.com).
\end{abstract}

Abstract

Keywords

- executive function

- cognitive function

- chronic kidney failure

- dialysis
Background Executive functions (EFs) are critical to daily life and sensitive to our physiological functioning and emotional states. The number of people living with chronic kidney disease (CKD) on hemodialysis (HD) globally is increasing steadily. We aimed to determine the impact of a single session of $\mathrm{HD}$ on EFs in patients with CKD receiving maintenance $\mathrm{HD}$ (MHD).

Methods This was a quasi-experimental study conducted at the department of psychiatry and dialysis unit of a tertiary hospital. Patients undergoing MHD underwent screening to rule out delirium, using the Confusion Assessment Method prior to EF testing. The tests of EF used were the Trail-Making Test-Part B (TMT-B) and Frontal Assessment Battery (FAB), both of which were administered before and after a session of HD. Statistical tests used were Wilcoxon matched pairs signed ranks test, paired $t$-test, single sample $t$-test, and correlation analyses.

Results The mean time taken on TMT-B before HD was 195.36 seconds and after HD, 171.1 seconds; difference is significant $(p=0.0001)$. The mean FAB score was 13.19 before HD and 14.83 after HD; the difference is significant $(p<0.0001)$. Significant differences were observed on similarities $(p=0.003)$, lexical fluency $(p=0.02)$, and go-no go $(p=0.003)$ subtests of FAB. Mean TMT-B scores before and after HD differed significantly from that of a reference study (reference TMT-B 150.69 seconds), $p=0.0002$ and 0.04 , respectively.

Conclusion We conclude that patients with CKD on MHD, in general, have worse executive cognitive functioning compared with healthy populations. A session of HD results in significant improvement in these functions.

\section{Introduction}

Neurological adverse outcomes like strokes or cognitive impairments, including dementias, encephalopathy, and delirium, are well documented in chronic kidney disease (CKD. ${ }^{1}$ Kidney transplantation, the gold-standard treatment of CKD, is cost-effective compared with hemodialysis (HD) and positively impacts patients' lives. Despite this, a large proportion of patients with CKD depend on dialysis due to paucity of organs, infrastructure, and trained human resource.

Executive functions (EFs) are central cognitive processes controlling problem-solving and resulting in goal-directed behavior. EFs include inhibitory control over prepotent responses, mental set-shifting, planning, monitoring and regulation of performance, and working memory. ${ }^{2}$ Abilities central to independent living like cooking, medication management, shopping, managing finances, transportation,
DOI https://doi.org/ 10.1055/s-0040-1703961 ISSN 0976-3147.
License terms

(요 (1) $\Theta \circledast$ 
laundry, housekeeping, and use of means of communication depend on preserved EFs. EFs are extremely sensitive to adverse milieu, induced, for example, by short term sleep deprivation and psychological stress. ${ }^{3-5}$ As metabolic parameters are known to fluctuate frequently over days or even hours in CKD, cognitive functions are expected to be affected adversely in this population.

Deficits in set-shifting or cognitive flexibility, sequencing, and inhibition of prepotent response are predictive of functional status in the nondemented elderly. ${ }^{6,7}$ Longer duration of CKD is associated with poor performance on tests of visual vigilance and inhibitory control., ${ }^{89}$ Children with CKD have demonstrated significant improvements in mental processing speed, working memory, stimulus discrimination, and reaction time after renal transplantation..$^{10}$

Dialysis reduces uremia, but at the cost of independence, with greater frailty and mortality. With long-term dialysis, noncompliance (missing scheduled dialysis sessions) due to inability to afford it, distance required to travel, inadequate health education, and depressive disorders is a recognized problem with serious consequences. ${ }^{11-13}$

Although dialysis initiation itself is associated with worsened cognitive performances, the immediate cognitive consequences of a session of dialysis remain relatively unexplored. ${ }^{14,15}$ We attempted to determine the effect of a single session of HD on EFs using simple bedside tests.

\section{Subjects and Methods}

This was a quasi-experimental study conducted by the department of psychiatry at the dialysis unit of a tertiary hospital attached to a medical college after Institutional Ethics Committee approval. Patients diagnosed with CKD undergoing maintenance HD (MHD) or those recently initiated on MHD at the time of study, and familiar with English alphabet and numbers, were included. Patients receiving dialysis for acute kidney injury and those with impaired comprehension, acute illness, medical instability, altered sensorium/delirium, active severe psychotic disorders, and any condition hindering the ability to use limbs effectively were excluded.

Sociodemographic and relevant medical information were recorded using a semi-structured questionnaire. Objective testing was done at two times-once before dialysis and then after completion of dialysis. Confusion Assessment Method was used to exclude delirious or confused patients before and after dialysis, prior to the administration of tests of EF. ${ }^{16}$

The following tests of executive functioning were conducted:

- Frontal Assessment Battery $(\mathrm{FAB})^{17}$ : It is used as a bedside tool to evaluate frontal lobe EFs like conceptualization (similarities), mental flexibility (lexical fluency), programming (motor series "Luria" test), sensitivity to interference (conflicting instructions), inhibitory control (go-no go test), and environmental autonomy (prehension behavior), with inter-rater reliability of 0.87 and internal consistency (Cronbach's coefficient $\alpha$ ) of 0.78 . Maximum possible score is 18 , with higher scores indicating better executive functioning. Some of the items in the FAB were adapted keeping in mind the general sociocultural background of our subjects (e.g., the subtest of lexical fluency).

- Trail-Making Test-Part B (TMT-B) ${ }^{18}$ : It is a paper-pencil test of cognitive flexibility that utilizes working memory. It requires the subject to connect circles containing a mixture of alphabet and numbers that have been randomly arranged. The subject is instructed to connect alphabet alternately with number in serial order. Performance is scored in time taken to complete the connection. There are no norms available for Indian population. The average time taken to complete the trail is $\sim 75$ seconds in primarily English-speaking populations.

Forty subjects were recruited over 2 months. One of the subjects refused to perform the TMT-B, but performed the FAB. Hence, analysis of performances on TMT-B were done using $n=39$. The FAB subtest scores of three subjects were lost during data compilation. Hence, the FAB performance was analyzed using $n=37$. Remaining analysis was done using $n=40$. Statistical tests used were Wilcoxon matched pairs signed ranks test (nonparametric alternative to the paired $t$-test), paired $t$-tests, and single sample $t$-test, and correlation analyses were done where appropriate.

\section{Results}

The sociodemographic information is tabulated in - Table 1 . From medical records, mean duration of CKD and MHD was 42.248 and 33.821 months, respectively. Hypertension was the most common comorbidity ( $87.5 \%)$, followed by diabetes mellitus (DM) 1 and 2 (20\%) and iron-deficiency anemia (10\%). Thirteen participants endorsed using nicotinecontaining products, 3 endorsed alcohol use, while 26 denied any substance use in the preceding 12 months.

Table 1 Descriptive statistics: sociodemographic data

\begin{tabular}{|l|l|}
\hline Variable & Distribution \\
\hline $\begin{array}{l}\text { Mean age in years } \\
(\text { SD) }\end{array}$ & 46.675 (14.967) \\
\hline $\begin{array}{l}\text { Gender distribution: } \\
n(\%)\end{array}$ & Males: 27 (67.5) \\
\cline { 2 - 2 } & Females: 13 (32.5) \\
\hline \multirow{3}{*}{ Marital status: $n$ (\%) } & Unmarried: 5 (12.5) \\
\cline { 2 - 2 } & Married: 31 (77.5) \\
\cline { 2 - 2 } & Widow/Widower: 4 (10) \\
\hline \multirow{5}{*}{ Education: $n$ (\%) } & No formal schooling: 3 (7.5) \\
\cline { 2 - 2 } & Up to standard X: 25 (62.5) \\
\cline { 2 - 2 } & Up to standard XII: 6 (15) \\
\cline { 2 - 2 } & Graduation and above: 6 (15) \\
\hline \multirow{3}{*}{ Occupation: $n$ (\%) } & $\begin{array}{l}\text { Unemployed/not working: } \\
\text { 25 (62.5) }\end{array}$ \\
\cline { 2 - 2 } & Employed: 15 (37.5) \\
\hline Family structure: $n$ (\%) & Living alone: 3 (7.3) \\
\cline { 2 - 2 } & Living with family: 37 (92.5) \\
\hline
\end{tabular}

Abbreviation: SD, standard deviation.

Note: Data computed for $n=40$.

ancludes housewives, retirees, and unemployed individuals. 
The findings of FAB and TMT-B testing are provided in - Tables 2 and 3. Statistically significant improvement was noted in performance after a session of HD with respect to average FAB scores, some of its subtests (similarities, lexical fluency, go-no go), and time taken to complete the trail test (-Table 4).

As no normative Indian data are available, we compared mean TMT-B score before and after HD in our study with those of a reference study conducted in North India on 120 healthy individuals. ${ }^{19}$ The mean time taken by this reference population was 150.69 seconds, which is below the accepted cutoff of 91 seconds that was used as a reference in their study. Our subjects' mean TMT-B before and after HD were 195.36 and 171.69 seconds, respectively. The difference between our scores and that of the reference study is statistically significant.

Correlation between duration of CKD and duration of MHD and the mean performances on each test and subtests were evaluated. The only significant findings were negative correlations between duration of CKD and mean TMT-B score $\left(r=-0.3816 ; P_{2}=0.02\right)$ and between duration of MHD and prehension subtest of FAB $\left(r=-0.4029 ; P_{2}=0.013\right)$. No significant correlation was found between the duration of CKD and MHD and remaining test scores.

\section{Discussion}

The mean age of our subjects was 46.6 years and 25 subjects were not working at the time of the study or were unemployed or housewives. Hence, almost $62 \%$ potentially employable people were not fulfilling this ability. Males are typically the bread-winners in our society and women supplement household income by working in fields in agricultural areas like the site of our study. About $48 \%(n=13)$ males were unemployed/not working.

Unemployment among a large sample of CKD patients in the Center for Cardiometabolic Risk Reduction in South Asia (CARRS) study was $6.2 \%$, or $n=670$ out of a total of 10,797 subjects from Chennai and Delhi. ${ }^{20}$ Mean age of the sample in this study ranged from 40.4 to 45.8 years, which is similar to our findings. CARRS included patients with CKD in all stages-not all were undergoing MHD, unlike our subjects who were all either already on MHD or had been initiated on MHD during the study. The process of HD and the number of working days it affects, limits occupational activities. Also, the setting of CARRS was urban, indicating more likelihood of finding gainful employment within easy traveling distances, variety of employment opportunities, and ease of obtaining

Table 2 Descriptive statistics: FAB and its subtests

\begin{tabular}{|c|c|c|c|c|c|}
\hline Test & Mean score & Mean $_{(1-I I)}$ & SD & 95\% Cl (lower limit) & 95\% Cl (upper limit) \\
\hline 1. $\mathrm{FAB}(\mathrm{I})$ & 13.19 & \multirow[t]{2}{*}{-1.64} & 2.60 & 12.32 & 14.05 \\
\hline 2. $\mathrm{FAB}(\mathrm{II})$ & 14.83 & & 2.33 & 14.05 & 15.61 \\
\hline 3. Mean FAB & 14.01 & - & 2.35 & 13.22 & 14.8 \\
\hline 4. $\quad$ Similarities (I) & 2 & \multirow[t]{2}{*}{-0.46} & 0.82 & 1.72 & 2.27 \\
\hline 5. Similarities (II) & 2.46 & & 0.6 & 2.25 & 2.66 \\
\hline 6. Lexical fluency $(\mathrm{I})$ & 1.65 & \multirow[t]{2}{*}{-0.3} & 0.79 & 1.38 & 1.91 \\
\hline 7. Lexical fluency (II) & 1.95 & & 0.85 & 1.66 & 2.22 \\
\hline 8. $\quad$ Motor series $(\mathrm{I})$ & 2.67 & \multirow[t]{2}{*}{-0.14} & 0.7 & 2.43 & 2.91 \\
\hline 9. Motor series (II) & 2.81 & & 0.57 & 2.62 & 3.00 \\
\hline 10. Conflicting instructions (I) & 2.13 & \multirow[t]{2}{*}{-0.22} & 1.00 & 1.8 & 2.47 \\
\hline 11. Conflicting instructions (II) & 2.35 & & 0.86 & 2.06 & 2.64 \\
\hline 12. Go-no go (I) & 1.78 & \multirow[t]{2}{*}{-0.49} & 0.91 & 1.48 & 2.09 \\
\hline 13. Go-no go (II) & 2.27 & & 0.93 & 1.96 & 2.58 \\
\hline 14. Prehension (I) & 2.97 & \multirow[t]{2}{*}{-0.03} & 0.16 & 2.91 & 3.02 \\
\hline 15. Prehension (II) & 3 & & 0 & 3 & 3 \\
\hline
\end{tabular}

Abbreviations: $\mathrm{Cl}$, confidence interval; FAB, Frontal Assessment Battery; SD, standard deviation.

Note: Series labeled "I" are before hemodialysis and "II" are after hemodialysis; $n=37$; mean FAB = average of FAB I and II.

Table 3 Descriptive statistics: Trail-Making Test-Part B (TMT-B)

\begin{tabular}{|c|c|c|c|c|c|}
\hline Test & $\begin{array}{l}\text { Mean score } \\
\text { (time in s) }\end{array}$ & Mean $_{(I-I I)}$ & SD & $\begin{array}{l}95 \% \mathrm{Cl} \\
\text { (lower limit) }\end{array}$ & $\begin{array}{l}95 \% \mathrm{Cl} \\
\text { (upper limit) }\end{array}$ \\
\hline 1. TMT-B (I) & 195.36 & \multirow[t]{2}{*}{23.67} & 68.08 & 173.28 & 217.44 \\
\hline 2. TMT-B (II) & 171.7 & & 62.87 & 151.31 & 192.08 \\
\hline 3. Mean TMT-B & 183.52 & - & 62.08 & 163.69 & 203.66 \\
\hline
\end{tabular}

Abbreviations: $\mathrm{Cl}$, confidence interval; SD, standard deviation; TMT-B, Trail-Making Test-Part B.

Note: Series labeled "I" are before hemodialysis and "II" are after hemodialysis; $n=39$; mean TMT-B = average of TMT-B I and II. 
HD within cities. Our study was almost exclusive to a rural population, where employment is mostly physically strenuous farm work, or jobs requiring tedious journeys to nearby towns, etc. These are hindered by the poor physical health of the patient and the resources expended on undergoing MHD in rural areas (in terms of time, money, energy, manpower). A study from Sao Paolo reported $44.44 \%$ of patients undergoing dialysis were either unemployed or not working due to retirement or sick leave. ${ }^{21}$ It is clear that CKD especially with MHD decreases employability and occupational productivity of patients and consequently reduces healthcare affordability. Further, $77.5 \%$ of our subjects were married and $92.5 \%$ were living with family suggesting that the majority of them had some social support. The Sao Paolo study also reported similar findings: $91.4 \%$ of their patients on dialysis were living with a family or friend. Family support provides alternative earning members, human resource to aid in activities of daily living, obtaining access to healthcare, etc.

The mean duration of CKD was 42.4 months $( \pm 40.4)$ and mean duration of MHD was 33.8 months $( \pm 25.6)$. Two or more chronic comorbid medical ailments were recorded in 18 (45\%) subjects. People with CKD undergoing MHD often suffer from multiple comorbid medical conditions that also require medical care in their individual capacity. ${ }^{22}$ This obviously adds to the financial burdens and reduces quality of life in this group. Nicotine was the most commonly used substance in our subjects in the preceding 12 months $(n=13)$, followed by alcohol $(n=3)$; 26 subjects reported no substance use in this period.

Performance on FAB in toto showed improvement after HD (Mean I - Mean II $=-1.64)$, which is significant $\left(P_{2}=<0.0001\right.$ ). In fact, the scores on the six subtests of FAB showed improvement from before to after HD. Improvements were significant for similarities, lexical fluency, and go-no go subtests $\left(P_{2}=0.003,0.02\right.$, and 0.003, respectively; - Table 4). These subtests prominently measure cognitive abilities like conceptual thinking, mental flexibility, and inhibitory control. The subtest that appeared to be least affected by HD was prehension-a test of inhibitory control to environmental cues (environmental autonomy).

The difference in mean time taken to complete TMT-B before and after HD was 23.67 seconds, which is significant $\left(P_{2}=0.0001\right)$. TMT-B specifically measures executive controlcognitive flexibility, working memory, and psychomotor speed; and our findings show improvement after a session of HD. ${ }^{19}$ On comparing our findings with that of a reference study using the single sample $t$-test, our subjects had a mean score of 195.36 seconds before HD, which is significantly more ( 150.69 seconds in reference study, $P_{2}=0.0002$ ). After $\mathrm{HD}$, the time taken reduced to 171.69 seconds, still significantly more than the reference $\left(P_{2}=0.04\right)$. The improvement in TMT-B performance after HD approached the average time taken by a healthy Indian population.

One study was found that addressed cognitive function before and after a single session of HD. ${ }^{23}$ In this study of 28 CKD patients on HD, significant improvements were observed in functions like logical and visual memories, memory quotient, psychomotor speed (using TMT-B), activity planning (a component of EF), and concentration, after a single session of HD. This study used an extensive battery of neuropsychological tests that, although desirable at research level, is difficult to execute routinely in clinical settings. We used simpler bedside tools that do not require extensive personnel training or equipment and are less time-consuming making them practical. A valuable lesson from such studies is that the day of dialysis is perhaps not the best time to communicate important information (like change in prescription, treatment strategy, etc.) to the patients as their processing ability is likely to be hampered. Most patients on MHD see their physicians on the day of, rather than the day after, dialysis. In

Table 4 Comparison of performance on tests of executive functions before and after hemodialysis

\begin{tabular}{|c|c|c|c|c|c|}
\hline \multicolumn{6}{|l|}{ A. } \\
\hline Test & $\begin{array}{l}\text { Sample } \\
\text { size }(n)\end{array}$ & w & $z$ & $\mathrm{~ns} / \mathrm{r}$ & $P_{2}$ \\
\hline 1. $F A B$ & 37 & -451 & -4.41 & 31 & $<0.0001^{\mathrm{a}}$ \\
\hline \multicolumn{6}{|l|}{ 2. Subtests of FAB } \\
\hline Similarities & \multirow[t]{4}{*}{37} & -171 & -2.96 & 21 & $0.003^{a}$ \\
\hline Lexical fluency & & -88 & -2.26 & 16 & $0.02^{\mathrm{a}}$ \\
\hline Conflicting instructions & & -68 & -1.47 & 18 & 0.14 \\
\hline Go-no go & & -159 & -2.96 & 20 & $0.003^{\mathrm{a}}$ \\
\hline 3. TMT-B & 39 & 545 & 3.8 & 39 & $0.0001^{\mathrm{a}}$ \\
\hline \multicolumn{6}{|l|}{ B. } \\
\hline Subtests of FAB & $\begin{array}{l}\text { Sample } \\
\text { size (n) }\end{array}$ & Mean $_{(I-I I)}$ & $\mathrm{t}$ & df & $P_{2}$ \\
\hline 1. Motor series & \multirow[t]{2}{*}{37} & -0.1351 & -1.3 & 36 & 0.20 \\
\hline 2. Prehension & & -0.027 & -1 & 36 & 0.32 \\
\hline
\end{tabular}

Abbreviations: FAB, Frontal Assessment Battery; TMTB, Trail-Making Test-Part B.

aSignificant; test used: Wilcoxon matched pairs signed rank test (for nonparametric data); paired $t$-test. 
a state of relative cognitive dysfunction at this time, they can, at least theoretically, be expected to have trouble planning and executing healthful behaviors. Scheduling such communication on the day after dialysis and including at least one family member in the process may be useful alternatives.

Duration of CKD and MHD both appeared to correlate positively with FAB scores (as duration of CKD or MHD increased, FAB scores improved), but it was not statistically significant $\left(P_{2}=0.14\right.$ and 0.53 , respectively). Similarly, duration of CKD and MHD showed a negative correlation with time taken on TMT-B $\left(P_{2}=0.02\right.$ and 0.25 , respectively). We found an improvement in performance on the trails test and FAB as the duration of CKD or MHD increased. The average time from diagnosis of CKD to initiation of MHD in our subjects was $\sim 8.4$ months (standard deviation \pm 30.6 ). Being a predominantly rural setup, with poor access to health care, most people do not seek medical attention early and it is very likely that patients received a diagnosis in advanced disease. Therefore, our finding of improvement in EF with increase in duration of disease is probably more reflective of the impact of maintenance dialysis.

Frequency of dialysis impacts survival in CKD, with thrice-weekly dialysis having better survival benefit than twice-weekly dialysis. ${ }^{24}$ In our study, $75 \%$ of the subjects were receiving dialysis twice-weekly, which is not unexpected in our part of the world. ${ }^{25}$ Previously discussed hindrances of cost and travel result in economy of dialyzing that not only reduces survival, but also affects quality of life. Patients on MHD often attempt to skip a dialysis session to save expenditure and seek dialysis when experiencing distressing symptoms like edema or breathlessness. ${ }^{11,12}$ We can see that this skipping can cost them their cognitive faculties, independence, and survival time. Since dialysis is found to improve cognitive functioning, increased frequency of dialysis to thrice-weekly can be postulated to be beneficial. In this context, peritoneal dialysis (PD) offers clients the advantages of ease of dialyzing at home, decreased need to travel for dialysis, and increased freedom to participate in occupational or social activities. Additionally, patients with end-stage renal disease on PD have lower risk for dementia compared with those on HD. ${ }^{26}$ Increasing use of PD can also reduce the infrastructural and human resource costs borne for setting up dialysis centers, making it an extremely viable option in areas with poor nephrology and renal replacement therapy services. ${ }^{27}$

The tests we used themselves are not extremely complex and the interval between assessments is usually between 3 and 5 hours. Hence, learning may explain some of the improvement we have observed. To mitigate this, use of relatively complex tasks or increasing the gap between assessments may be suitable. When looking into the effect of a single session of dialysis, it is difficult to achieve a reasonable gap in assessments.

Our findings provide evidence for need to optimize dialysis frequency, conduct routine bedside cognitive evaluations, include a caregiver during nephrology/physician consultations to improve treatment adherence, and engage in healthful behaviors in CKD patients.

\section{Conclusion}

We conclude that patients with CKD on MHD, in general, have worse executive cognitive functioning compared with healthy populations. A session of HD results in significant improvement in these functions compared with predialysis performances.

\section{Funding}

The study was conducted under the Short Term Studentship (STS) program by the Indian Council of Medical Research (ICMR) awarded to second author under the guidance of the first author in 2018-2019.

\section{Conflict of Interest}

V.S.M. reports grants from Indian Council of Medical Research-Short Term Studentship 2018-19, during the conduct of the study.

\section{References}

1 Arnold R, Issar T, Krishnan AV, Pussell BA. Neurological complications in chronic kidney disease. JRSM Cardiovasc Dis 2016;5:2048004016677687

2 Diamond A. Executive functions. Annu Rev Psychol 2013;64:135-168

3 Vartanian O, Bouak F, Caldwell JL, et al. The effects of a single night of sleep deprivation on fluency and prefrontal cortex function during divergent thinking. Front Hum Neurosci 2014;8:214

4 Tucker AM, Whitney P, Belenky G, Hinson JM, Van Dongen HP. Effects of sleep deprivation on dissociated components of executive functioning. Sleep 2010;33(1):47-57

5 Starcke K, Wiesen C, Trotzke P, Brand M. Effects of acute laboratory stress on executive functions. Front Psychol 2016;7:461

6 Jefferson AL, Paul RH, Ozonoff A, Cohen RA. Evaluating elements of executive functioning as predictors of instrumental activities of daily living (IADLs). Arch Clin Neuropsychol 2006;21(4):311-320

7 Bell-McGinty S, Podell K, Franzen M, Baird AD, Williams MJ. Standard measures of executive function in predicting instrumental activities of daily living in older adults. Int J Geriatr Psychiatry 2002;17(9):828-834

8 Mendley SR, Matheson MB, Shinnar S, et al. Duration of chronic kidney disease reduces attention and executive function in pediatric patients. Kidney Int 2015;87(4):800-806

9 Fennell EB, Fennell RS, Mings E, Morris MK. The effects of various modes of therapy for end stage renal disease on cognitive performance in a pediatric population-a preliminary report. Int J Pediatr Nephrol 1986;7(2):107-112

10 Mendley SR, Zelko FA. Improvement in specific aspects of neurocognitive performance in children after renal transplantation. Kidney Int 1999;56(1):318-323

11 Griva K, Lai AY, Lim HA, Yu Z, Foo MW, Newman SP. Non-adherence in patients on peritoneal dialysis: a systematic review. PLoS One 2014;9(2):e89001

12 Ibrahim S, Hossam M, Belal D. Study of non-compliance among chronic hemodialysis patients and its impact on patients' outcomes. Saudi J Kidney Dis Transpl 2015;26(2):243-249

13 Kutner NG, Zhang R, McClellan WM, Cole SA. Psychosocial predictors of non-compliance in haemodialysis and peritoneal dialysis patients. Nephrol Dial Transplant 2002;17(1):93-99

14 Post JB, Jegede AB, Morin K, Spungen AM, Langhoff E, Sano $\mathrm{M}$. Cognitive profile of chronic kidney disease and hemodialysis patients without dementia. Nephron Clin Pract 2010;116(3):c247-c255 
15 Kurella Tamura M, Vittinghoff E, Hsu CY, et al; CRIC Study Investigators. Loss of executive function after dialysis initiation in adults with chronic kidney disease. Kidney Int 2017;91(4):948-953

16 Inouye SK, van Dyck CH, Alessi CA, Balkin S, Siegal AP, Horwitz RI. Clarifying confusion: the confusion assessment method. A new method for detection of delirium. Ann Intern Med 1990;113(12):941-948

17 Dubois B, Slachevsky A, Litvan I, Pillon B. The FAB: a frontal assessment battery at bedside. Neurology 2000;55(11): 1621-1626

18 Reitan RM. Validity of the Trail Making Test as an indicator of organic brain damage. Percept Mot Skills 1958;8(3):271-276

19 Bhatia T, Shriharsh V, Adlakha S, Bisht V, Garg K, Deshpande SN. The trail making test in India. Indian J Psychiatry 2007;49(2):113-116

20 Anand S, Shivashankar R, Ali MK, et al; CARRS Investigators. Prevalence of chronic kidney disease in two major Indian cities and projections for associated cardiovascular disease. Kidney Int 2015;88(1):178-185

21 Cruz MC, Andrade C, Urrutia M, Draibe S, Nogueira-Martins LA, Sesso RdeC. Quality of life in patients with chronic kidney disease. Clinics (São Paulo) 2011;66(6):991-995
22 Lakshminarayana GR, Sheetal LG, Mathew A, Rajesh R, Kurian G, Unni VN. Hemodialysis outcomes and practice patterns in end-stage renal disease: experience from a tertiary care hospital in Kerala. Indian J Nephrol 2017;27(1):51-57

23 Schneider SM, Malecki AK, Müller K, et al. Effect of a single dialysis session on cognitive function in CKD5D patients: a prospective clinical study. Nephrol Dial Transplant 2015;30(9):1551-1559

24 Chandrashekar A, Ramakrishnan S, Rangarajan D. Survival analysis of patients on maintenance hemodialysis. Indian J Nephrol 2014;24(4):206-213

25 Jha V. Current status of end-stage renal disease care in India and Pakistan. Kidney Int Suppl 2013;3(2):157-160

26 Wolfgram DF, Szabo A, Murray AM, Whittle J. Risk of dementia in peritoneal dialysis patients compared with hemodialysis patients. Perit Dial Int 2015;35(2):189-198

27 Abraham G, Varughese S, Mathew M, Vijayan M. A review of acute and chronic peritoneal dialysis in developing countries. Clin Kidney J 2015;8(3):310-317 